\title{
Field validation of a semi-spherical Lagrangian drifter*
}

\author{
M. GASSER, J. SALVADOR, P. SANGRÀ and J.L. PELEGRÍ \\ Departamento de Física, Facultad de Ciencias del Mar, Universidad de Las Palmas de Gran Canaria, \\ 35017 Las Palmas de Gran Canaria, Canary Islands, Spain.
}

\begin{abstract}
SUMMARY: We present the design and field validation for the performance of a Semi-spherical Lagrangian Drifter (SELD). The SELD is a low-cost, low-technology, home-made alternative to much more expensive commercial Lagrangian drifters. An analysis of the SELD configuration shows that its drag area ratio is as good as TRISTAR and holey-sock drifters. Field results in a situation of near solid rotation within an oceanic vortex confirm that SELD and holey-sock drifters have
\end{abstract} an almost identical behavior.

Key words: Lagrangian drifters, drifter design, drag area ratio, oceanic vortex.

\section{INTRODUCTION}

The water-following performance of drifters has been traditionally studied either by measuring or modeling the forces acting on the drifter (e.g. Kirwan et al., 1975, 1978; Vachon, 1980; Chhabra, 1985; Chereskin et al., 1989; Krauss et al., 1989), or through the measurement of the relative slip between the drifter and the surrounding waters (e.g. Niiler et al., 1987, 1995; Geyer, 1989). In the first approach the resulting force experienced by the drifter may be measured with a dynamometer at different towing speeds, or modeled after careful examination of the shapes and drag coefficients for the different parts configuring the buoy system. In the second one the drifter is typically instrumented with Vector Measuring Current Meters (VMCM) and the statistics of the current series are compiled.

\footnotetext{
*Received March 10, 2000. Accepted July 28, 2000.
}

A third approach has been to compare the behavior of different types of drifters or to compare the currents inferred from the drifter motion with velocity directly measured from fixed current meters. Previous works in this direction have either examined the dispersion of drifters initially deployed close to each other (Kirwan et al., 1978; Mackas et al., 1987) or compared the trajectory of a drifter with the vector diagram that would be derived from the current meter data at a nearby fixed position (McPhaden et al., 1991). One limitation of this approach is the potentially very different behavior that the drifters may experience (or the drifter and the current meter) once they get sufficiently apart. This is actually the case in near-coastal regions where the currents vary rapidly depending on coastal morphology. Hence we would be forced to deploy the drifters in horizontally homogeneous dynamical regions: far from the coast and away from any frontal region, with near homogeneous wind fields and mixed layer depths. Such a situation is probably rare, or at least hard to verify. 
A different alternative would be to deploy the drifters in conditions where a current regime is dominant. These conditions may arise in regions of strong inertial oscillations, such as in the surface mixed layer (but recall the difficulty of verifying wind and mixed layer homogeneity), or intense tidal currents, such as in elongated estuaries. Even in these cases, however, the spatial variability may be large enough to obscure the validity of the intercomparison. A better case for our purposes would be eddies undergoing near solid rigid rotation. Such structures are rather common features in the ocean, usually generated after the current detaches from a coastal region or island, or following the unstable growth of a frontal meander. These structures have been reported by several authors as rather permanent features, and in some cases have been tracked using drifters (Gould, 1985; Glenn et al., 1990; Pingree and Le Cann, 1991, 1992; Arístegui et al., 1994; Pingree, 1996, 1997).

Glenn et al. (1990) deployed drifters in Gulf Stream rings and found that these are in approximate solid body rotation up to a radius of about $25 \mathrm{~km}$. Pingree and Le Cann (1991) deployed one drifter in the Northeast Atlantic which spent 66 days in a cyclonic eddy and 130 days in an anticyclonic one. In the cyclonic eddy the orbits were often elliptical or even egg-shaped but the period of rotation showed little dependence on the radius (up to $40 \mathrm{~km}$ ). In the anticyclonic eddy the trajectories were almost per- fectly circular and the inner part $(<5 \mathrm{~km})$ was in approximate solid rotation. Pingree and Le Cann (1991) also showed that despite the presence of surface winds or currents the drifter could remain trapped within the eddy with no translation tendency.

The above characteristics are quite ideal for our purpose of intercomparison of drifter behavior and are the motivation for this paper. We first describe the configuration of a Semi-Spherical Lagrangian Drifter (SELD) with its drogue at $100 \mathrm{~m}$ depth. We next calculate the drag area ratio $R$ (Geyer, 1989; Niiler et al., 1995) and compare its value to those for the TRISTAR and holey-sock drifters. This is a first indicator that suggests that the behavior of the SELD, TRISTAR and holey-socked drifters should be analogous. We end up comparing the behavior of the SELD and holey-sock drifters when deployed within an anticyclonic eddy, generated by the presence of Gran Canaria Island on the path of the Canary Current (for a description of these eddies see Aristegui et al., 1994). For this comparison the three drifters are deployed along a radial axis, with the SELD drifter in a central position in between the two holey-socked drifters.

\section{BUOY CONFIGURATION}

The SELD consists of three surface small buoys and the subsurface semi-circular dragging drogue.
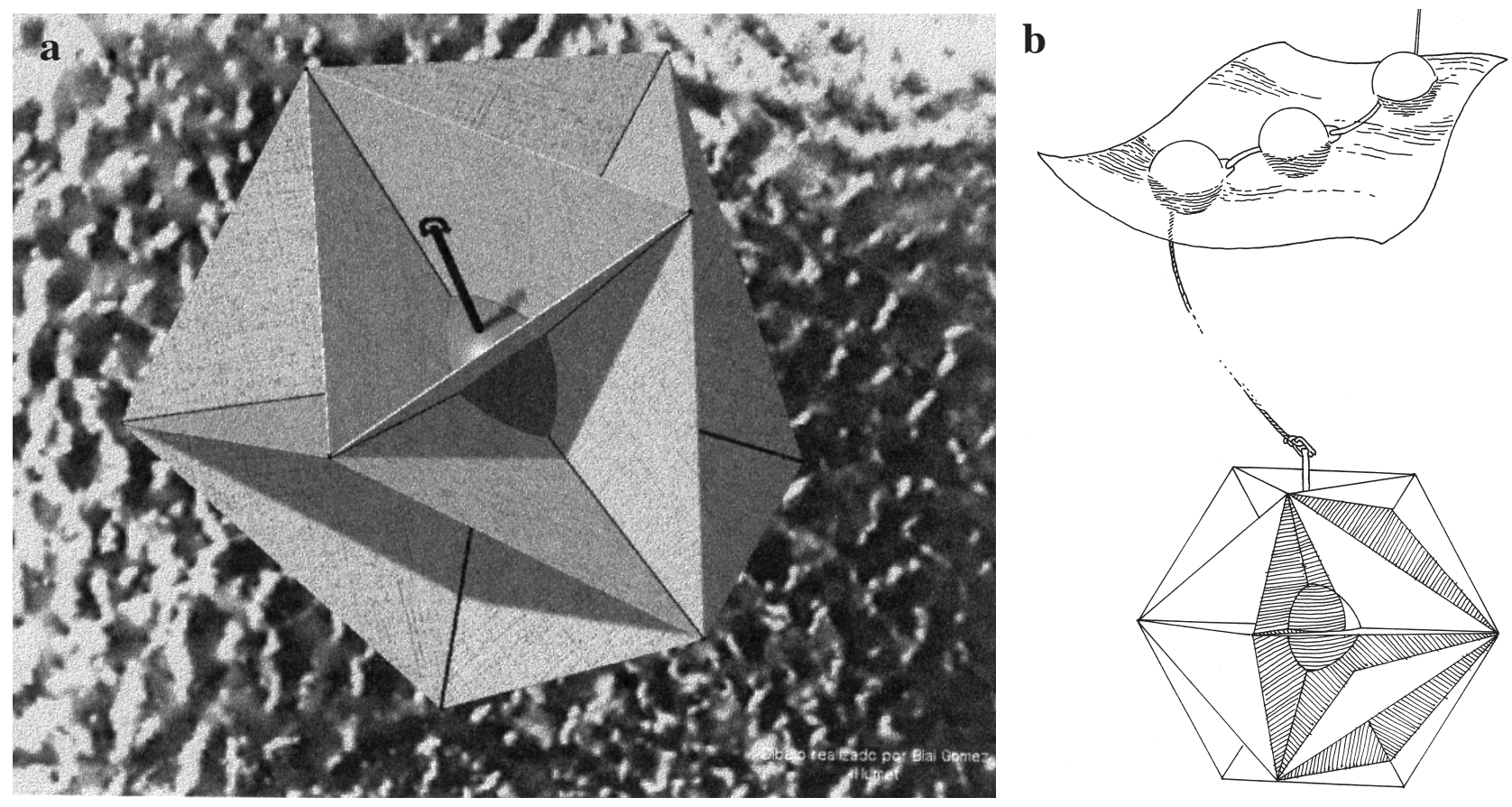

FIG. 1. - Schematics of drifter configuration: (a) view of SELD's drogue, (b) perspective view of buoy system 
The surface buoys are spherical with $0.5 \mathrm{~m}$ diameter, one of them containing the ARGOS positioning system and the other two providing buoyancy to the system. The drogue is tethered to the third surface buoy with a $100 \mathrm{~m}$ long nylon rope $(0.8 \mathrm{~cm}$ diameter). The drogue has a sea urchin like configuration, with 1.4 $\mathrm{m}$ long PVC standard tube (0.05 $\mathrm{m}$ diameter) arms protruding from a $0.5 \mathrm{~m}$ diameter sphere. Connections between the sphere and the tubes were made from standard plumbing PVC unions. A trapezoidal canvas is tied between the arms, providing an extensive drag surface. Except the central buoy, the SELD is made from pieces available from a hardware store. The prototype was manufactured by one of the authors in a few days, with the help of several undergraduate students, its cost being a small fraction of commercial drifters. The SELD can be mounted by three untrained people in less than half an hour.

Figure 1a presents a schematic of the drogue and Figure $1 \mathrm{~b}$ shows a perspective view of the buoy system. The density of PVC is slightly larger than the water density making the system sink to nearly the full extent of the tether. The density difference, however, is small enough to allow the three top buoys to remain in the sea surface, avoiding unnecessary strength between the surface buoys and the tether line. Otherwise, tension between the surface buoys and the drifter was not measured. Figure 2 presents a photograph of SELD on board of the ship, the compactness of the drogue makes its deployment a rather simple maneuver.

\section{CALCULATION OF DRAG AREA RATIO}

The movement of a lagrangian drifter differs from the motion of the water mass where it is located due to the tension exerted by the tethering rope. This tension arises from the vertical velocity gradient in the ocean. Generally, the surface buoy velocity due to waves and currents is much higher than the drifter velocity. Therefore its drag forces will be different, this difference being what produces a relative force on the drifter that modifies its motion.

Drag forces are equal to the projected area of the element times the water velocity times the drag coefficient. As much as the ratio between the drag on the drifter and the external drag is enough large, the drifter movement will be due to the water mass influence rather than to external forces. We can estimate the difference between the drifter velocity and the water mass velocity as a function of that ratio.

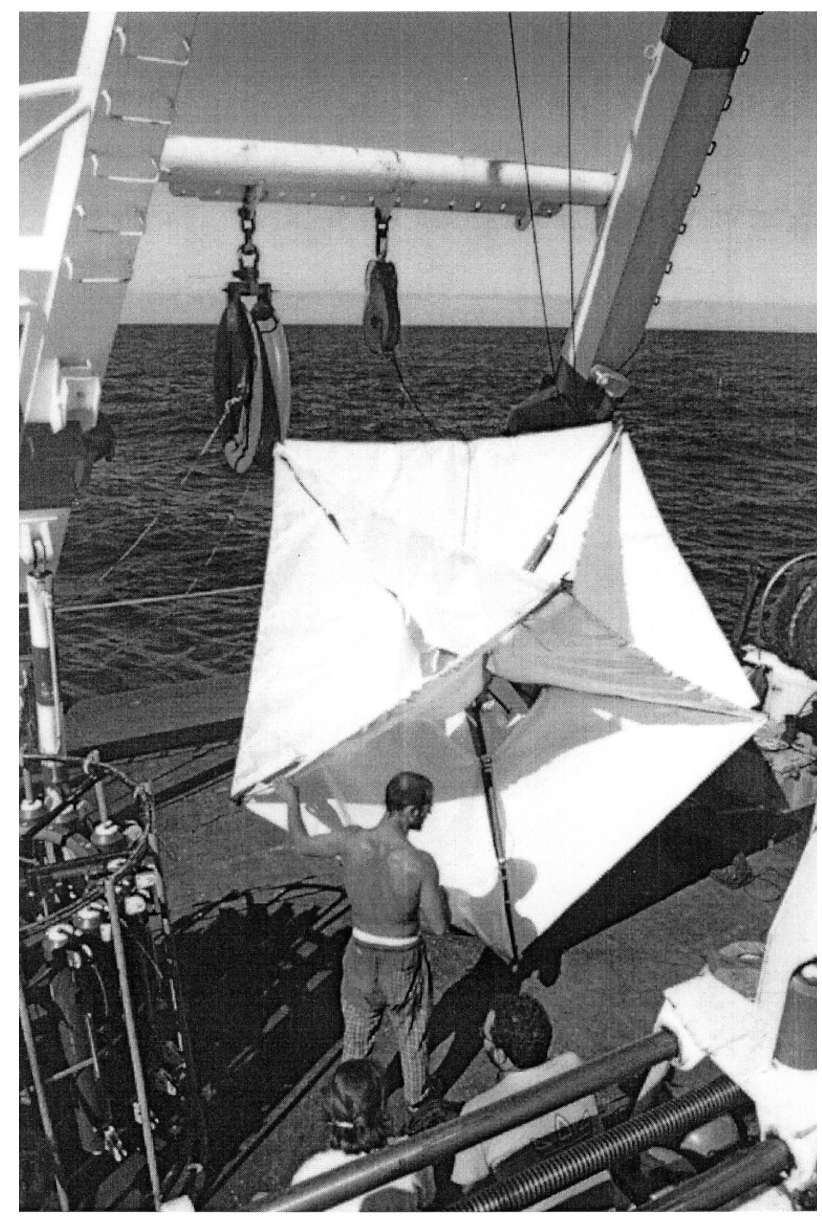

FIG. 2. - Photograph of SELD just before deployment in the water.

Let us define the drag area ratio $R$ (Niiler et al., 1995) as:

$$
R=\frac{C_{d} A_{d}}{\sum C_{s} A_{s}}
$$

In this equation $C$ is the drag coefficient and $A$ the projected area. The $s$ and $d$ subindexes are used to indicate the surface elements (buoys, rope) and drifter, respectively.

The desired velocity difference, or slip velocity $U$, may be estimated from wind data and the vertical gradient of the current velocity according to the following relationship:

$$
U_{s}=a R^{-1} U_{w}+b R^{-1} \Delta U
$$

where $U_{w}$ is the wind velocity and $\Delta U$ is the vertical velocity gradient. The capability of the drifter to follow the water movements increases when the slip velocity is small.

Table 1 presents the areas, drag coefficients, drag areas, and drag area ratio for SELD when tethered to 
TABLE 1. - Dimensions, areas, drag coefficients, drag areas, and drag area ratios for SELD tethered to 10 and $100 \mathrm{~m}$ depths.

\begin{tabular}{|c|c|c|c|c|c|}
\hline & Dimensions & Area $\left(\mathrm{m}^{2}\right)$ & $C$ & $C A$ & $R=C_{d} A_{d} / \Sigma C_{s} A_{s}$ \\
\hline Surface buoys (x 3) & $0.50 \mathrm{~m} \varnothing$ & 0.20 & 0.5 & $3 \times(0.5 \times 0.2)=0.3$ & \\
\hline \multirow[t]{2}{*}{ Rope } & $0.01 \mathrm{~m} \varnothing, 10 \mathrm{~m}$ length case & 0.10 & 1 & $1 \times 0.1=0.1$ & $11.97 /(0.3+0.1)=29.92$ \\
\hline & $0.01 \mathrm{~m} \varnothing, 100 \mathrm{~m}$ length case & 1.00 & 1 & $1 \times 1=1$ & $11.97 /(1+0.1)=10.88$ \\
\hline Drifter & $3.30 \mathrm{~m} \varnothing$ & 8.55 & 1.4 & $1.4 \times 8.55=11.97$ & \\
\hline
\end{tabular}

$10 \mathrm{~m}$ and to $100 \mathrm{~m}$ depths. The SELD drag coefficient was estimated as that for half a sphere with its open end facing the flow. We have used $10 \mathrm{~m}$ depth in order to compare our SELD with TRISTAR and holey-sock values reported in the literature. We obtain $R=30$ for a SELD drifter tethered at $10 \mathrm{~m}$, which is a value similar to those reported by Niiler et al. (1995) for the TRISTAR and holey-sock drifters. When the SELD drifter is tethered at a depth of $100 \mathrm{~m}$ we obtain $R=11$, a relative large value. With the coefficients from Niiler et al. (1995), a $5 \mathrm{~m} \mathrm{~s}^{-1}$ wind and $\Delta U=10^{-3} \mathrm{~s}^{-1}$ we obtain a slip velocity $U_{s}=0.02 \mathrm{~m} \mathrm{~s}^{-1}$

\section{FIELD TESTING}

In order to test the behavior of the SELD drifter we deployed one SELD and two holey-socked drifters in one of the very energetic vortex which are generated by the impinging of the Canary Current on the Canary Archipelago (Arístegui et al., 1994).
In order to avoid contamination by differential drifting in the near-surface mixed layer the drifters were deployed with a tether of $100 \mathrm{~m}$. This causes a relatively small drag area ratio $(R=11)$, so we expect the water-following capabilities of the drifters to be somehow diminished.

The three drifters were deployed approximately along a radial section of the vortex, with the SELD drifter in between the two holey-socked drifters. Figure $3 \mathrm{a}$ illustrates the trajectories of the three drifters during about two days, shortly after their deployment within an anticyclonic vortex south of Gran Canaria island. Figure $3 b$ indicates the position where the drifters were launched and illustrates the temperature distribution across the vortex taken immediately before the drifters' deployment. The anticyclonic character of the vortex is indicated by the presence of relatively warm water in the central region (Arístegui et al., 1994).

Figure 3 a suggests that both the period of rotation of the drifters and the separation between them is rather coherent in time, with all three buoys a

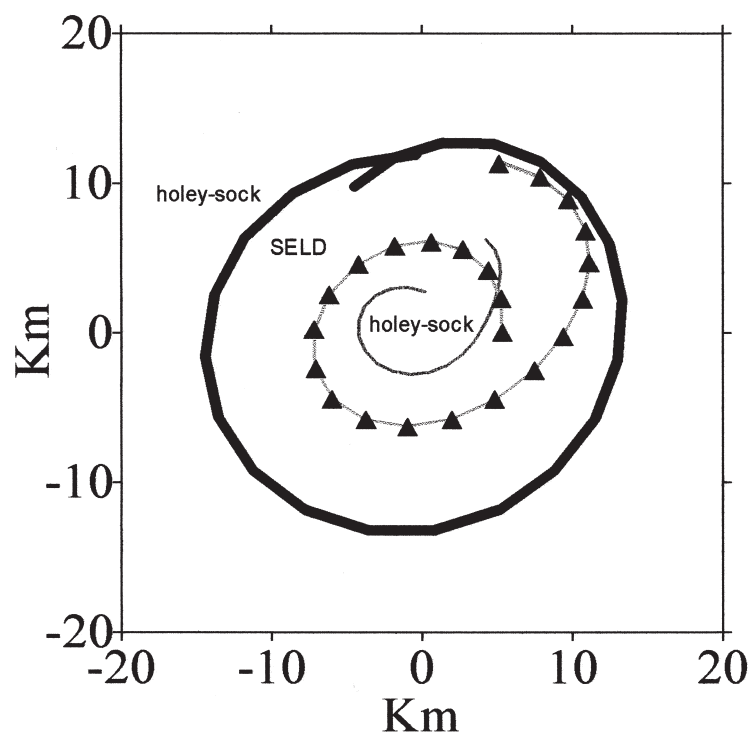

b

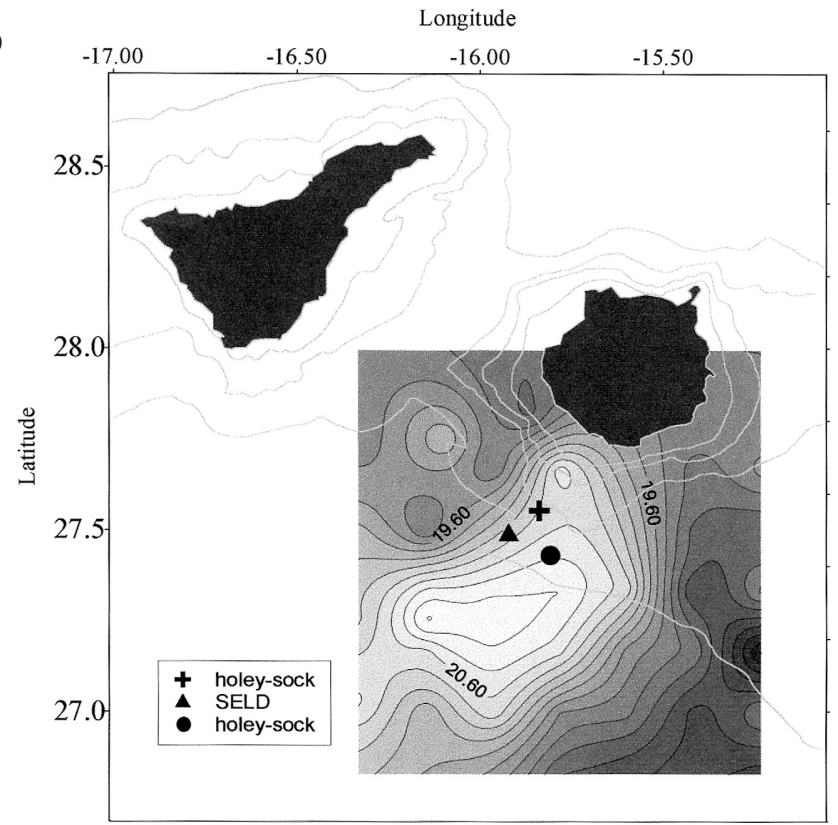

FIG. 3. - (a) Buoy trajectories of one SELD and two holey-socked drifters during about two days, tick marks for the SELD buoy are every two hours. (b) Surface temperature distribution in the experiment zone previous to the deployment of the buoys. 


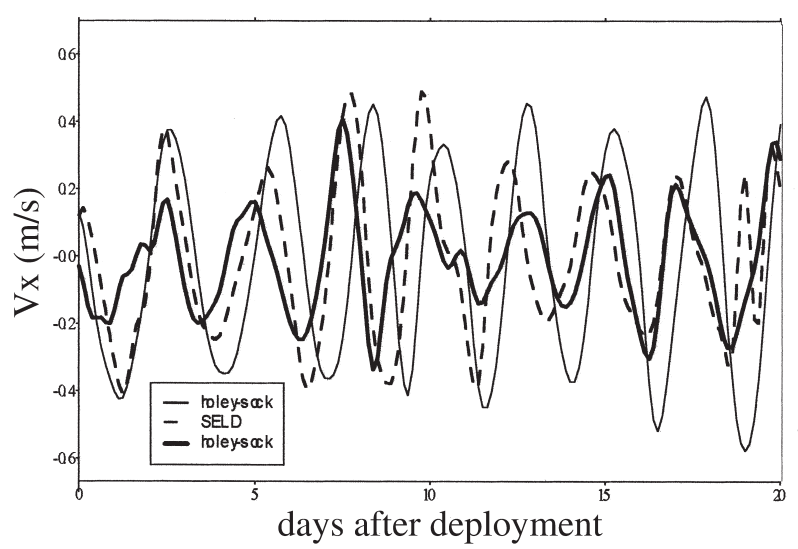

FIG. 4. - East-west velocities for all three buoys during the first 20 days after deployment (positive values correspond to eastward velocities).

rotating in a motion very close to solid body rotation. This is confirmed by Figure 4, which presents the east-west velocities during the first 20 days after deployment: the period is maintained and any phase difference is related to the fact that the deployment was not exactly along a radial section of the vortex.

The SELD drifter showed to be a very strong buoy, its transmission lasted during about 150 days. In fact it ceased transmitting shortly after one holey-socked drifter and before the other one. We believe that the durability of the SELD buoy is due to its compactness and we expect that in the future, after minor modifications, it may be further improved.

\section{CONCLUSIONS}

The buoy configuration, the calculation of its drag area ratio $(R)$, and a novel field test for a semispherical lagrangian drifter (SELD) are presented. The drag area ratio of the SELD drifter is similar to that of standard holey-socked drifters, its field behavior being analogous to the behavior of holeysocked drifters. The main advantage of the drifter is its compactness and durability.

The tethering at $100 \mathrm{~m}$ depths has the disadvantage of greatly reducing the drag area ratio but has the advantage of avoiding contamination in the surface layer by movements different from vortex near-solid rotation. The limitations could be important if the buoys were deployed in low-velocity flows but they show to be of very little importance in the very energetic anticyclonic vortex south of Gran Canaria island.

\section{ACKNOWLEDGEMENTS}

We would like to sincerely thank both Oswaldo López-Monzón and Simón Ruiz for a number of very useful comments. This research has been supported by the Spanish government through projects FRENTES (CYCIT's grant number AMB950731) and TALUD (CYCIT's grant number MAR96-1893), and by the European Union through project CANIGO (MAST's grant number MAS3-CT96-0060).

\section{REFERENCES}

Arístegui, J., P. Sangrà, S. Hernández-León, M. Cantón, A. Hernández-Guerra and J. Kerling. - 1994. Island-induced eddies in the Canary Islands. Deep-Sea Res., 42: 1509-1525.

Chereskin, T.K., P.P. Niiler and P.M. Poulain. - 1989. A numerical study of the effect of upper-ocean shear on flexible drogued drifters. J. Atmos. Oceanic Tech., 6: 243-152.

Chhabra, N.K. - 1985. Low cost drifter analytical design study. The Charles Stark Draper Laboratory, Cambridge, MA, CSDL-R$1781,43 \mathrm{pp}$.

Geyer, W.R. - 1989. Field calibration of mixed layer drifters. $J$. Atmos. Oceanic Tech., 27: 443-456.

Glenn, S.M., G.Z. Forristall, P. Cornillon and G. Milkowski. 1990. Observations of Gulf Stream ring 83-E and their interpretation using feature models. J. Geophys. Res., 95: 1304313063.

Gould, W.J. - 1985. Physical oceanography of the Azores Front. Progress Oceanogr., 14: 167-190.

Kirwan, A.D., Jr., G. McNally, M.S. Chang and R. Molinari. 1975. The effect of wind and surface currents on drifters. $J$. Phys. Oceanogr., 5: 361-368.

Kirwan, A.D., Jr., G. McNally and S. Pazan. - 1978. Wind drag and relative separation of undrogued drifters. J. Phys. Oceanogr., 8: 1146-1150.

Krauss, W., J. Dengg and H.H. Hinrichsen. - 1989. The response of drifting buoys to currents and wind. J. Geophys. Res., 94: 32013210 .

Mackas, D.L., W.R. Crawford and P.P. Niiler. - 1987. Comparisons of trajectories of two Lagrangian drifters. J. Atmos. Oceanic Tech., 27: 443-456.

McPhaden, M.J., D.V. Hansen and P.L. Richardson. - 1991. A comparison of ship drift, drifting buoy and current meter mooring velocities in the Pacific South Equatorial Current. J. Geophys. Res., 96: 775-782.

Niiler, P.P., R.E. Davis and H.J. White. - 1987. Water-following characteristics of a mixed layer drifter. Deep-Sea Res., 31: 1867-1881.

Niiler, P.P., A.S. Sybrandy, K. Bi, P.M. Poulain and D. Bitterman. - 1995. Measurements of the water-following capability of holey-sock and TRISTAR drifters. Deep-Sea Res., 42: 1951-1964.

Pingree, R.D. - 1996. A shallow subtropical subducting westward propagating eddy (Swesty). Philos. Trans. Royal Soc. London, 354. 979-1026.

Pingree, R.D. - 1997. The eastern subtropical gyre (North Atlantic): flow rings recirculations structure and subduction. J. Mar. Biol. Assoc. U.K., 77: 573-624.

Pingree, R.D., and B. Le Cann. - 1991. Drifting buoy in the field of flow of two eddies on East Thulean Rise (Northeast Atlantic). J. Geophys. Res., 96: 16759-16777.

Pingree, R.D., and B. Le Cann. - 1992. Anticyclonic eddy X91 in the southern Bay of Biscay, May 1991 to February 1992. $J$. Geophys. Res., 97: 14353-14367.

Vachon, W.A. - 1980. Drifters. In: F. Dobson (ed.), Air-Sea Interaction: Instruments and Methods, pp. 201-218. Plenum, New York. 
